\title{
Démarche qualité en médecine: un état des lieux par portraits succincts (13)
}

\footnotetext{
www.fmh.ch $\rightarrow$ Welcome $\rightarrow$ Qui sommes-nous? $\rightarrow$ Le Secrétariat général $\rightarrow$ Données, démographie \& qualité
}

La démarche qualité en médecine s'est établie dans notre pays. C'est ce que le groupe de travail Qualité de la FMH (GTQ-FMH) tient à montrer aux membres de la FMH. Il présente ainsi dans cinq numéros consécutifs du BMS les initiatives Qualité qui lui ont été annoncées depuis 2006 par des médecins de toutes les disciplines médicales et de tous les secteurs d'activité (domaine hospitalier/ambulatoire). Ces descriptifs sont également publiés sur le site internet de la FMH, Département Données, démographie et qualité avec des informations pratiques telles que graphiques ou bibliographie. * La présente édition est consacrée au questionnaire de la communauté de travail «Satisfaction des patients» ARPAZ.

\section{Questionnaire de la communauté de travail «Satisfaction des patients» ARPAZ}

\author{
Pour le lecteur pressé \\ Le questionnaire sur la satisfaction des pa- \\ tients de I'ARPAZ \\ ... répond au principe que seule une critique libre \\ de préjugés amène le changement (esprit cri- \\ tique et non pas une culture du jugement) \\ ... garantit l'anonymat grâce au recours à une \\ entreprise externe \\ ... permet au médecin d'avoir des éléments de \\ comparaison avec ses collègues, sans la pres- \\ sion d'un classement ou d'un impératif à \\ $s$ 'améliorer \\ ... existe en allemand et en français \\ ... a un effet positif sur les patients qui, grâce à \\ ce questionnaire, se sentent pris au sérieux
}

\section{Description}

En 1993, sous l'égide de l'Institut d'économie d'entreprise de l'Ecole polytechnique de Zurich et en étroite collaboration avec les patients, l'Association des internistes zurichois a élaboré un questionnaire relatif à la satisfaction des patients: en toute indépendance vis-àvis des médecins, les patients ont formulé des questions et les ont pondérées. Le questionnaire est proposé par la communauté de travail «Satisfaction des patients (ARPAZ)». La philosophie à la base du document est que seule une critique libre de préjugés amène le changement (esprit critique et non pas une culture du jugement).

Sur la base d'une comparaison avec ses collègues, le médecin peut identifier ses forces et faiblesses et modifier sa manière de faire; il n'y a néanmoins pas de classement ni d'impératif à s'améliorer. Le questionnaire a aussi un impact positif sur les patients. Ils sentent que leur opinion compte et sont donc d'autant plus motivés à remplir le questionnaire avec soin.

Le questionnaire de cette évaluation volontaire comprend 27 questions ainsi qu'une rubrique pour les commentaires. Une évaluation comprend 150 questionnaires; les patients envoient leurs questionnaires anonymisés directement à une entreprise externe qui est responsable de l'évaluation. Le médecin participant reçoit le dossier d'évaluation de même que le duplicata des appréciations librement formulées par les patients.

\section{Aptitude \\ En priorité pour les cabinets de médecine de premier re- cours; compte tenu de l'ampleur de la base de données disponibles, il existe la possibilité d'adapter le ques- tionnaire pour d'autres spécialités.}

\section{Charge en temps}

Par patient; saisie globale; modifications selon les réponses et à l'initiative du praticien concerné.

\section{Charge financière}

Le prix total pour une évaluation s'élève à Fr. 700.Si le mandataire le souhaite, le prix peut être baissé à Fr. 300.- en recourant à un sponsoring externe. Le sponsor n'a accès à aucune information relative aux résultats ni au mandataire.

Modifications selon les réponses et à l'initiative du praticien concerné.

\section{Informations complémentaires}

ARPAZ et Quali Doc. Primary Care 2002;2:157-163

www.zuercher-internisten.ch $\rightarrow$ Der VZI $\rightarrow$ Evaluation der Patientenzufriedenheit 